\title{
COMPACTAÇÃO, DENSIDADE E FERTILIDADE DO SOLO NA ÁREA DE PRESERVAÇÃO PERMANENTE DO IGARAPÉ DOS TANQUES - PORTO VELHO/RO
}

Gabriel Ediu dos Santos Pereira $^{1}$, Marilia Locatelli ${ }^{2}$, Robison Costa de Souza ${ }^{3}$

${ }^{1}$ Mestrando em Geografia - Universidade Federal de Rondônia - UNIR

${ }^{2}$ Pesquisadora da Embrapa Rondônia e Profa. no Programa de Pós-Graduação em Geografia da UNIR (marilia.locatelli@embrapa.br)

${ }^{3}$ Mestrando em Geografia - Universidade Federal de Rondônia - UNIR

Recebido em: 08/04/2016 - Aprovado em: 30/05/2016 - Publicado em: 20/06/2016 DOI: 10.18677/Enciclopedia_Biosfera_2016_140

\begin{abstract}
RESUMO
Os solos das Áreas de Preservação Permanente - APP urbanas estão sob diferentes influências, o que pode levar a compactação. Nesse sentido foi realizado estudo na APP do Igarapé dos Tanques na cidade de Porto Velho - RO.Objetivouse com a realização deste estudo identificar a compactação do solo por meio da quantificação da resistência do solo à penetração - RP na APP da área de estudo e de investigar se esses resultados influenciam no crescimento das raízes. $O$ levantamento foi conduzido nos dias 22/01/2016 e 23/01/2016, onde foram realizadas avaliações de RP nas profundidades de $0-10 \mathrm{~cm}, 10-20 \mathrm{~cm}, 20-30 \mathrm{~cm}$ e $30-40 \mathrm{~cm}$, em oito pontos distintos, sendo o resultado composto da média de 5 repetições em cada ponto. Em todos os oito pontos avaliados, foi retirado uma amostra indeformada de solo para determinação da densidade e umidade. Foi realizada análise de fertilidade do solo em cada ponto. Os testes de RP foram realizados com penetrômetro de impacto. Verificou-se que em todos os oito pontos avaliados a RP ultrapassou o valor de 2,0 MPa. A densidade do solo variou de 1,28 a $1,84 \mathrm{~kg} \cdot \mathrm{m}^{-3}$. Em relação à fertilidade do solo, apenas o elemento $\mathrm{Ca}$ apresentou valores satisfatórios. Para os demais pontos algumas características químicas se apresentaram satisfatória e outras não, o que sugere a necessidade de adubação e/ou calagem diferenciada nos pontos avaliados para que as plantas tenham 0 desenvolvimento ideal.
\end{abstract}

PALAVRAS-CHAVE: Área de Preservação Permanente Urbana, Compactação do solo, Fertilidade do solo.

\section{COMPRESSION, DENSITY AND SOIL FERTILITY IN PERMANENT PRESERVATION AREA OF TANKS RIVER - PORTO VELHO/RONDÔNIA}

\begin{abstract}
The soils of the urban Permanent Preservation Areas - PPA are under different influences, which can lead to compression. In this sense the study was conducted in Igarapé dos Tanques PPA in the city of Porto Velho - Rondônia. The study aimed to identify soil compaction by quantifying soil penetration resistance in the PPA of the
\end{abstract}


study area and to investigate whether these results influence roots growth. The survey was conducted in the days 01.22.2016 and 01.23.2016, which were carried out soil resistance penetration ratings in the depths of $0-10 \mathrm{~cm}, 10-20 \mathrm{~cm}, 20-30 \mathrm{~cm}$ and $30-40 \mathrm{~cm}$ in eight distinct points, and the result being composed of the average of 5 replicates at each point. Ratings in all eight points evaluated one undisturbed soil sample for determination of moisture and density was collected. Soil fertility analysis was performed on each point. The soil penetration resistance tests were performed with an impact penetromete. It was found that in all eight points assessed resistance penetration exceeds the value of $2.0 \mathrm{MPa}$. The bulk density ranged from 1.28 to 1.84 $\mathrm{kg} \mathrm{m}^{-3}$. In relation to soil fertility, only the Ca element showed satisfactory values. For other points some chemical characteristics were satisfactory and not others, suggesting fertilization need and / or differentiated liming in points assessed so that plants can have optimal development.

KEYWORDS: Permanent Preservation Urban Area, Soil compaction, Soil fertility.

\section{INTRODUÇÃO}

Com o crescimento desordenado das cidades, os problemas ambientais são cada vez mais frequentes e refletem negativamente na qualidade de vida da população. Desta forma, as áreas urbanas se tornaram objetos de estudos em busca de soluções para problemas como deslizamento de solo, processos erosivos, alagamentos, degradação dos cursos d'água, altas temperaturas, etc (CAMPOS, et al., 2014). Neste contexto, as Áreas de Preservação Permanente - APP dos cursos d'água apresentam grande importância. Segundo o Código Florestal (BRASIL, 2012), para cursos d'água de menos de 10 (dez) metros de largura, deve-se respeitar a largura mínima de 30 (trinta) metros das faixas marginais. Área de Preservação Permanente trata-se de área protegida, coberta ou não por vegetação nativa, com função ambiental de preservar os recursos hídricos, a paisagem, a estabilidade geológica e a biodiversidade, facilitar o fluxo gênico de fauna e flora, proteger o solo e assegurar o bem-estar das populações humanas (BRASIL, 2012).

A resistência do solo à penetração - RP e a densidade do solo - DS são algumas das características físicas do solo que indica o grau de compactação e, consequentemente, a facilidade de penetração das raízes no solo (LIMA et al., 2013; ASSIS et al., 2014). A umidade do solo, além de fatores intrínsecos do solo (textura, estrutura e mineralogia), exerce influência na RP. Com o aumento da umidade do solo, diminui a atuação das forças de coesão entre as partículas do solo e o atrito interno, levando a redução da RP (KUNZ et al., 2013; GUBIANI et al., 2014).

Objetivou-se com a realização deste estudo verificar a compactação do solo em APP do Igarapé dos Tanques na área urbana do município de Porto Velho - RO por meio de penetrômetro de impacto, observando condições favoráveis ou não para o desenvolvimento de espécies arbóreas nativas destinadas a reflorestamento misto em APP, como por exemplo, Piquiá (Caryocar villlosum), Castanha-do-Brasil (Bertholletia excelsa), Cedro (Cedrella fissilis), Garapa (Apuleia leiocarpa), Paineira (Chorisia speciosa), Jatobá (Hymeniaea courbaril var. Stillocarpa), Paricá (Schizolobium amazonicum), Sumaúma (Ceiba pentandrea (L.) Gaertn) (SALMAN et al., 2008). Para isso foi realizado uma avaliação da compactação nos primeiros 40 $\mathrm{cm}$ de solo, verificando as zonas mais compactadas $(0-10 \mathrm{~cm}, 10-20 \mathrm{~cm}, 20-30$ $\mathrm{cm}, 30-40 \mathrm{~cm}$ ). Paralelo às informações de RP também foi realizado levantamento referente a umidade gravimétrica, densidade aparente e fertilidade do solo. 


\section{MATERIAL E MÉTODOS}

\section{Área de Estudo}

O presente estudo foi realizado na Área de Preservação Permanente do Igarapé dos Tanques que passa dentro da cidade de Porto Velho - RO. O Igarapé dos Tanques está inserido na Amazônia Ocidental, estado de Rondônia, região central da cidade de Porto Velho e pertence à bacia do rio Madeira. Desde a nascente até a foz, encontra-se totalmente inserido no núcleo urbano da cidade de Porto Velho, percorrendo uma distância total de quatro quilômetros e oitocentos e trinta metros $(4,83 \mathrm{~km})$.

A nascente do Igarapé dos Tanques está localizada no Bairro Nova Porto Velho e a foz na margem direita do Rio Madeira, no Bairro Nacional. Dentre a Área de Preservação Permanente compreendida pelo Igarapé dos Tanques, foram selecionados oito pontos para a avaliação da compactação, umidade, densidade e fertilidade do solo. Esses pontos estão localizados em seis bairros da cidade (Nova Porto Velho, Embratel, Liberdade, Olaria, São João Bosco e Costa e Silva) conforme exposto na Figura 1.

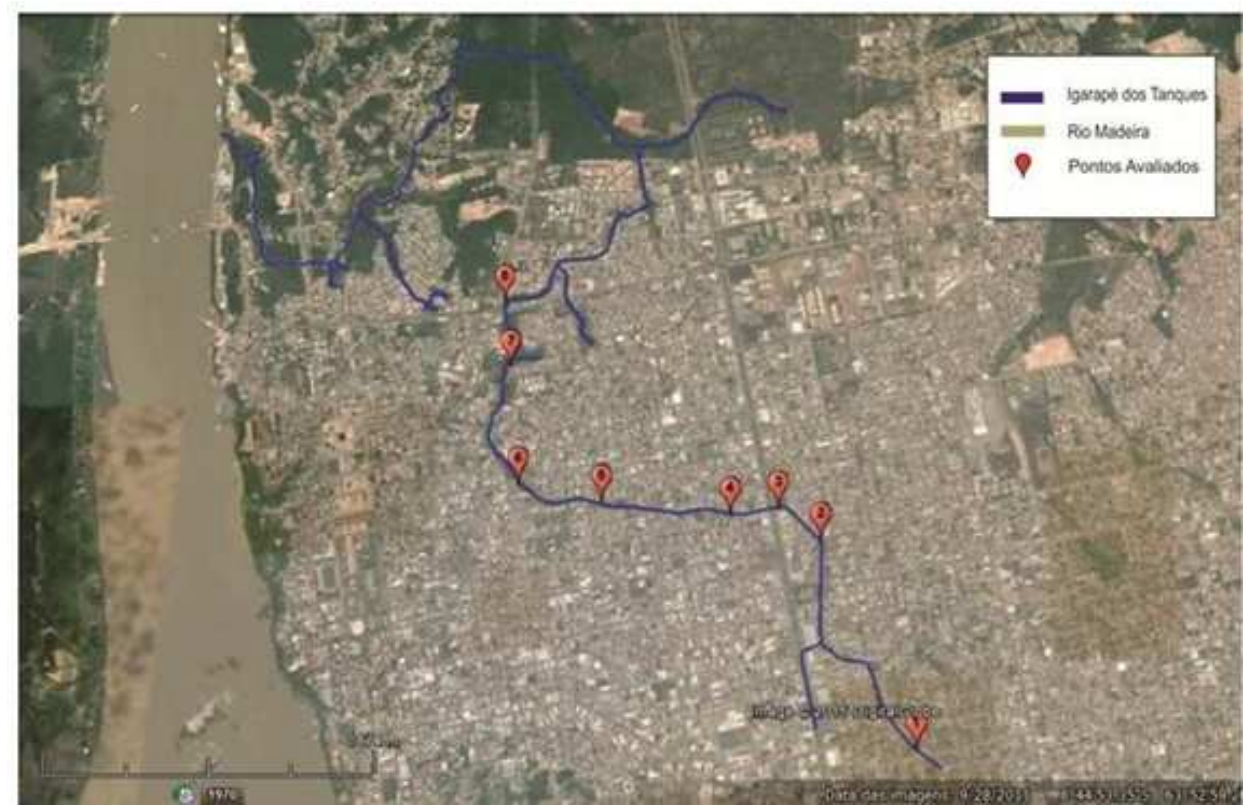

FIGURA 1. Distribuição dos pontos ao longo da APP do Igarapé dos Tanques.Fonte: Imagem Landsat Google Earth.

O Clima na região de Porto Velho, de acordo com a classificação de Köppen, é do tipo Awi - Tropical chuvoso com estação seca no ano. A temperatura média é de $26^{\circ} \mathrm{C}$, com máxima de $32^{\circ} \mathrm{C}$ e mínima de $21^{\circ} \mathrm{C}$. Em Porto Velho a precipitação média no período chuvoso, que compreende os meses de novembro a abril é superior a $220 \mathrm{~mm}$ por mês, já nos períodos secos, que compreende os meses de junho a agosto a média da precipitação diminui para $31,2 \mathrm{~mm}$ por mês, (RONDÔNIA, 2015).

De acordo com a Secretaria de Estado do Desenvolvimento Ambiental SEDAM (FERNANDES et al., 2001), o perímetro urbanizado do município de Porto Velho está assentado sobre Latossolo Vermelho-Amarelo, e há predominância de solos distróficos e álicos, ou seja, de baixa fertilidade natural e alta acidez. Vale ENCICLOPÉDIA BIOSFERA, Centro Científico Conhecer - Goiânia, v.13 n.23; p.1571 2016 
ressaltar que a caracterização do solo para a área de estudos corresponde ao solo originário, porém, atualmente com a ação antrópica causada pelo núcleo urbano da cidade de Porto Velho, na área de APP do Igarapé dos Tanques observou-se a deposição de entulho, lixo, aterro de diversos tipos, materiais que não correspondem mais ao solo originário.

\section{Coleta dos dados}

Para a coleta das amostras de solo e dos valores de RP foi delimitado um perímetro de $10 \times 20 \mathrm{~m}$ em cada um dos oito pontos. Dentro desse perímetro foram realizadas 5 repetições para a coleta dos dados em cada um dos pontos de estudo. Dessa forma foram realizadas 40 avaliações de RP nos oito pontos de estudo (Figura 2).

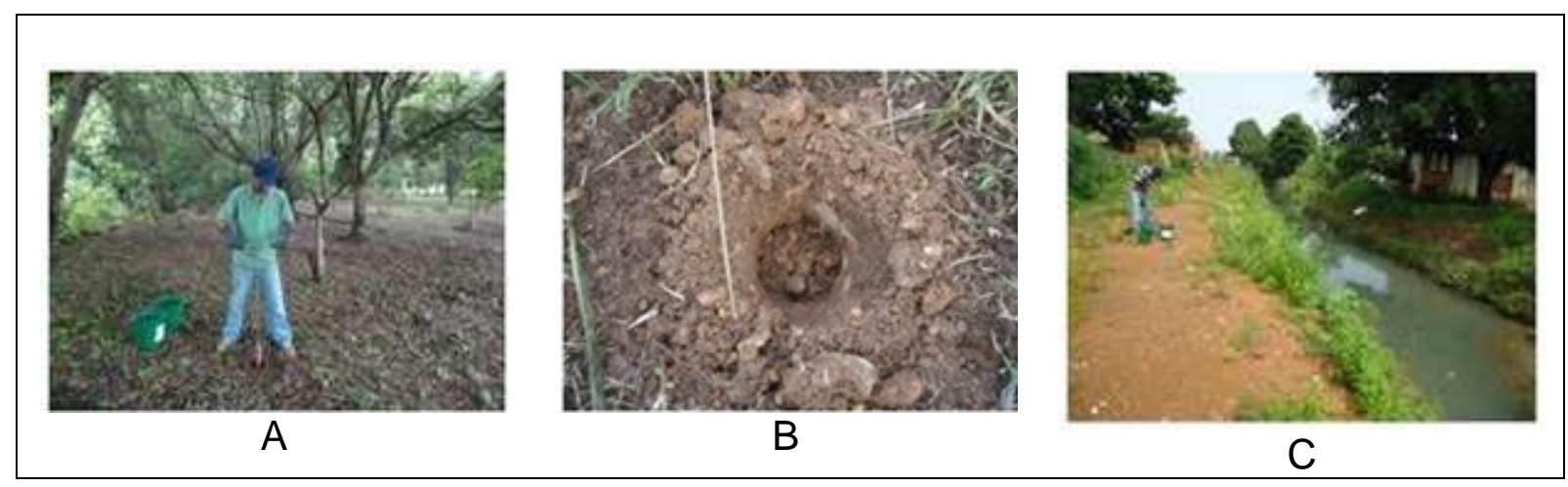

FIGURA 2 A) Coleta de solo; B) Um dos pontos de amostragem de solo (ponto 6); C) APP do Igarapé dos Tanques sob influência antrópica.Fonte: Os autores.

O levantamento dos dados foi conduzido nos dias 22/01/2016 e 23/01/2016, onde foram realizadas as avaliações de RP nas profundidades de $0-10 \mathrm{~cm}, 10-20$ $\mathrm{cm}, 20-30 \mathrm{~cm}$ e $30-40 \mathrm{~cm}$, nos oito pontos de estudo.

Em cada um dos oito pontos em que foram realizados os testes de RP, também foi retirada uma amostra indeformada de solo para determinação da umidade gravimétrica e da densidade do solo. Além disso, essas amostras foram submetidas à análise de fertilidade, conforme EMBRAPA (2011).

A RP foi determinada por meio do penetrômetro de impacto (IAA/PLANALSUCAR), conforme método descrito por STOLF et al. (1983). O número de impactos e profundidade de penetração obtidos em campo foram transformados em unidades de MPa utilizando o programa de computação de dados em Excel - VBA (STOLF, 2011). Os resultados de RP foram submetidos à análise de variância pelo teste $\mathrm{F}$ e as médias foram comparadas pelo teste de Tukey a $5 \%$ de probabilidade através do programa estatístico ASSISTAT versão 7.7 (SILVA, 2016). Os resultados de RP foram comparados com base em (BENGOUGH, MULLINS, 1990), onde foi considerado 2,0 MPa como limite crítico ao crescimento de raízes de espécies arbóreas nativas destinadas a reflorestamento misto da APP do Canal dos Tanques. 


\section{Compactação do solo}

A densidade do solo (DS) dos oito pontos de estudo apresentaram valores variando de 1,28 a $1,84 \mathrm{~kg} \cdot \mathrm{dm}^{-3}$ (Tabela 1). Para solos argilosos (> $35 \%$ argila) a DS considerada como crítica situa-se entre 1,30 a $1,40 \mathrm{~kg} \cdot \mathrm{dm}^{-3}$, para solos de textura média entre 1,40 a $1,50 \mathrm{~kg}^{-\mathrm{dm}^{-3}}$ e para solos de textura arenosa de 1,70 a 1,80 $\mathrm{kg} . \mathrm{dm}^{-3}$. A DS é influenciada pela textura, solos arenosos apresentam densidade superior ao solo argiloso, enquanto que os solos siltosos apresentam comportamento intermediário (LIBARDI, 2005).

TABELA 1. Valores de densidade e umidade gravimétrica do solo de estudos na Área de Preservação Permanente - APP do Igarapé dos Tanques, Porto Velho/RO.

\begin{tabular}{lcc}
\hline Local & Densidade $\left(\mathrm{kg} \cdot \mathrm{dm}^{-3}\right)$ & Umidade $\left(\mathrm{kg} \cdot \mathrm{kg}^{-1}\right)$ \\
\hline Ponto 1 & 1,28 & 0,20 \\
Ponto 2 & 1,83 & 0,11 \\
Ponto 3 & 1,84 & 0,05 \\
Ponto 4 & 1,73 & 0,14 \\
Ponto 5 & 1,51 & 0,12 \\
Ponto 6 & 1,68 & 0,09 \\
Ponto 7 & 1,58 & 0,18 \\
Ponto 8 & 1,60 & 0,17 \\
\hline
\end{tabular}

Quanto aos valores de umidade presentes na Tabela 1, o ponto 1 foi o que apresentou a maior quantidade de umidade no solo, $0,20 \mathrm{~kg} \cdot \mathrm{kg}^{-1}$, enquanto que o ponto 3 foi o que teve a menor umidade, $0,05 \mathrm{~kg} \cdot \mathrm{kg}^{-1}$. Os valores de densidade e umidade apresentam uma relação inversa, sendo que o ponto 1 apresentou maior umidade e menor densidade, enquanto que o ponto 3 apresentou menor umidade $e$ maior densidade. Estudos mostraram relação entre menor umidade e maior RP, sendo mais estreita a relação na faixa de menor umidade (BENEDETTI et al. 2010; LIMA et al., 2013).

Como indicativo da compactação do solo foi utilizado medidas de RP por meio de penetrômetro de impacto. Tais dados apresentam relações diretas com o desenvolvimento das plantas e são mais eficientes na identificação do estado de compactação quando comparados à densidade do solo (CONTE et al, 2011; SANTANA et al., 2014).

As medidas de RP na Área de Preservação Permanente do Igarapé dos Tanques, em diferentes profundidades, 0-10, 10-20, 20-30 e 30-40 cm, são apresentadas na Tabela 2. Os valores da RP encontrados ao longo da Área de Preservação Permanente do Igarapé dos tanques mostram que os solos da área em estudo então compactados em todas as 4 profundidades dos oito pontos avaliados, ou seja, estão acima do valor considerado crítico ao crescimento radicular de 2,0 MPa (BENGOUGH \& MULLINS, 1990), com exceção apenas da profundidade 0-10 $\mathrm{cm}$ do ponto 8 , que apresentou valor de $1,35 \mathrm{MPa}$, porém esse valor não apresenta diferença significativa em relação a outros pontos que encontram-se com valores de RP acima de 2,0 MPa na mesma profundidade (pontos 2, 3 e 7) e em relação a profundidade de $30-40 \mathrm{~cm}$ para o mesmo ponto (ponto 8). No ponto 8, diferentemente dos outros, foi observado que foram realizadas duas gradagens 6 meses antes da avaliação, o que pode explicar o valor menor de RP na 
profundidade $0-10 \mathrm{~cm}$ deste ponto. Os pontos que apresentaram as maiores resistências foram os ponto 2 e 3 nas profundidades de $20-30$ e 30-40 cm e o ponto 6 nas profundidades de 10-20, 20-30 e 30-40 cm.

TABELA 2. Resistência à penetração do solo (MPa) nos oito pontos de estudos na Área de Preservação Permanente -APP- do Igarapé dos Tanques, Porto Velho/RO.

\begin{tabular}{ccccccccc}
\hline Prof. & \multicolumn{7}{c}{ Resistência à penetração (Mpa) } \\
\cline { 2 - 9 }$(\mathrm{cm})$ & Ponto 1 & Ponto 2 & Ponto 3 & Ponto 4 & Ponto 5 & Ponto 6 & Ponto 7 & Ponto 8 \\
\hline $0-10$ & $3,42 \mathrm{aBC}$ & $2,49 \mathrm{bBC}$ & $4,09 \mathrm{bABC}$ & $4,41 \mathrm{aABC}$ & $5,47 \mathrm{aAB}$ & $6,83 \mathrm{aA}$ & $2,91 \mathrm{bBC}$ & $1,35 \mathrm{bC}$ \\
$10-20$ & $3,80 \mathrm{aCD}$ & $9,16 \mathrm{abABC}$ & $9,53 \mathrm{abAB}$ & $4,65 \mathrm{aBCD}$ & $7,19 \mathrm{aBCD}$ & $14,15 \mathrm{aA}$ & $4,64 \mathrm{aBCD}$ & $3,01 \mathrm{aD}$ \\
$20-30$ & $3,13 \mathrm{aBC}$ & $14,52 \mathrm{aA}$ & $10,12 \mathrm{abABC}$ & $4,31 \mathrm{aBC}$ & $7,43 \mathrm{aABC}$ & $12,30 \mathrm{aAB}$ & $4,21 \mathrm{abBC}$ & $2,55 \mathrm{aC}$ \\
$30-40$ & $5,67 \mathrm{aAB}$ & $10,54 \mathrm{abAB}$ & $12,92 \mathrm{aA}$ & $3,55 \mathrm{aAB}$ & $8,05 \mathrm{aAB}$ & $10,71 \mathrm{aAB}$ & $3,72 \mathrm{abAB}$ & $2,22 \mathrm{abB}$ \\
\hline
\end{tabular}

Médias seguidas pela mesma letra minúscula na coluna e maiúscula na linha não diferem estatisticamente entre si pelo Teste de Tukey a $5 \%$ de probabilidade.

A variação dos dados de resistência mecânica à penetração do solo em diferentes profundidades e entre os pontos de coleta é mais bem visualizada na Figura 3.

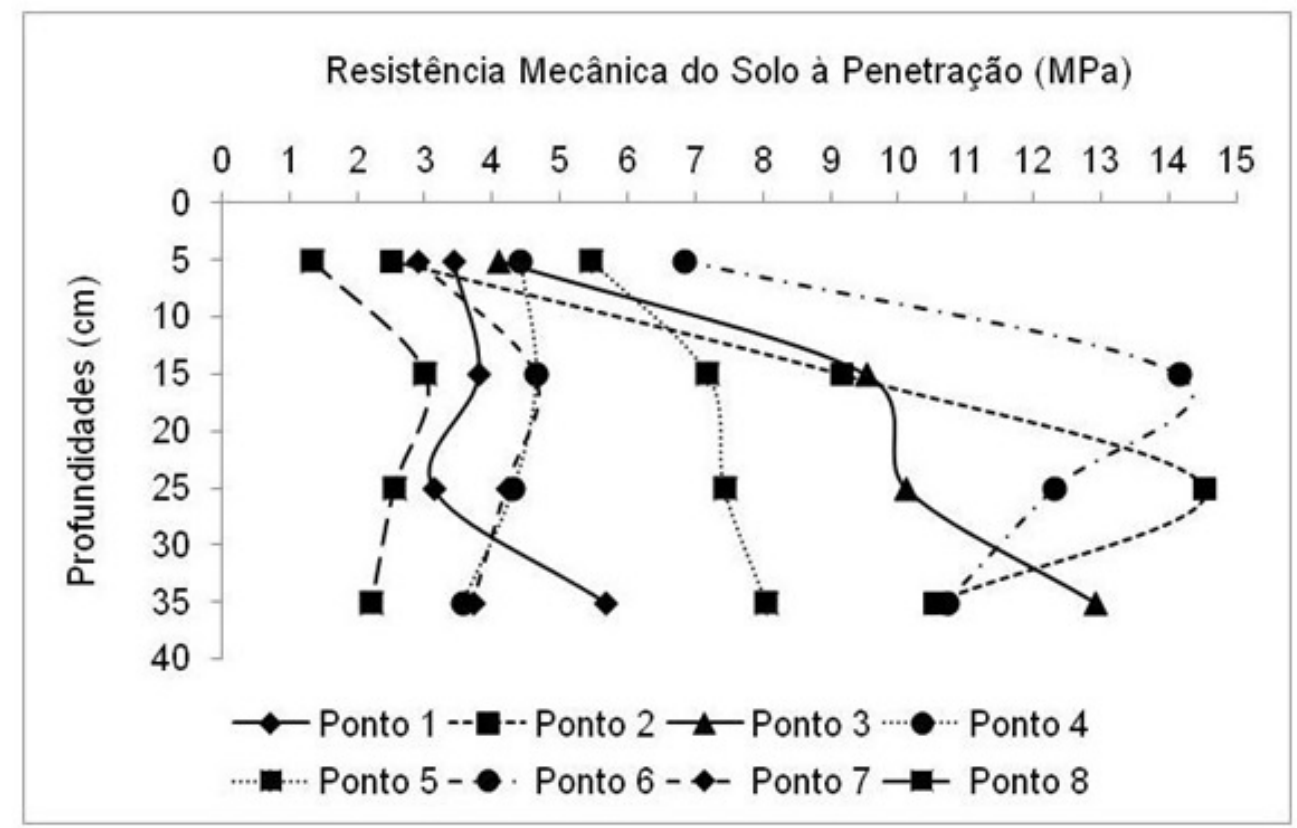

FIGURA 3 Resistência mecânica do solo à Penetração (MPa) em diferentes profundidades nos oito pontos de estudos na Área de Preservação Permanente - APP do Igarapé dos Tanques, Porto Velho/RO.

$\mathrm{Na}$ Figura 3 é possível observar que os pontos 2, 3 e 6 são os que apresentam as maiores resistências e os pontos 1, 4, 7 e 8 , os que apresentaram os menores valores de resistência.

Os pontos que apresentaram os maiores valores de RP são os mesmos que apresentaram os menores valores de umidade. Tal resultado está de acordo com GUBIANI et al. (2013), que afirmam que pequenas reduções na umidade 
proporcionam incrementos acentuados da RP. ORTIGARA et al., (2014) também afirmam que estudos experimentais têm mostrado que a RP aumenta com a elevação da densidade e diminui com a elevação do teor de água do solo.

\section{Fertilidade do solo}

Os resultados de fertilidade do solo dos oito pontos analisados na APP do Igarapé dos Tanques são apresentados na Tabela 3.

A interpretação da análise de fertilidade do solo foi realizada de forma que abrangesse variados tipos de espécies arbóreas nativas, já que a intenção é o reflorestamento misto de APP, assim sendo, os dados foram comparados com parâmetros gerais de adubação estimados por RIBEIRO et al. (1999) constantes nas tabelas 5 e 6.

TABELA 3. Resultado da análise de fertilidade do solo nos oito pontos de estudos na Área de Preservação Permanente - APP do Igarapé dos Tanques, Porto Velho/RO.

\begin{tabular}{|c|c|c|c|c|c|c|c|c|c|c|c|}
\hline \multirow{2}{*}{ Locais } & $\mathrm{pH}$ & MO & $P$ & $\mathrm{~K}$ & $\mathrm{Ca}$ & $\mathrm{Mg}$ & $\mathrm{H}+\mathrm{Al}$ & Al & СТС & $\mathrm{m}$ & V \\
\hline & Água & $\mathrm{g} \mathrm{kg}^{-1}$ & $\mathrm{mg} \mathrm{dm}^{-3}$ & \multicolumn{6}{|c|}{$\mathrm{cmol}_{\mathrm{c}} \mathrm{dm}^{-3}$} & \multicolumn{2}{|c|}{$\%$} \\
\hline Ponto $1(0-20 \mathrm{~cm})$ & 7,1 & 9,2 & 57 & 0,18 & 8,09 & 0,5 & 2 & 0 & 10,75 & 0 & 82 \\
\hline Ponto $1(20-40 \mathrm{~cm})$ & 6 & 5,8 & 11 & 0,11 & 5,47 & 0,33 & 3,5 & 0 & 9,37 & 0 & 63 \\
\hline Ponto $2(0-20 \mathrm{~cm})$ & 5,7 & 18,5 & 14 & 0,15 & 3,74 & 0,5 & 5,4 & 0 & 9,77 & 0 & 45 \\
\hline Ponto $2(20-40 \mathrm{~cm})$ & 6,2 & 12,3 & 24 & 0,12 & 4,75 & 0,33 & 3,7 & 0 & 8,94 & 0 & 58 \\
\hline Ponto $3(0-20 \mathrm{~cm})$ & 8 & 19,5 & 6 & 0,17 & 9,49 & 0,22 & 0,8 & 0 & 10,64 & 0 & 93 \\
\hline Ponto $3(20-40 \mathrm{~cm})$ & 8 & 12,6 & 7 & 0,11 & 8,93 & 0,19 & 0,6 & 0 & 9,84 & 0 & 94 \\
\hline Ponto $4(0-20 \mathrm{~cm})$ & 6,6 & 13,1 & 67 & 0,16 & 6,53 & 0,34 & 3,9 & 0 & 10,89 & 0 & 65 \\
\hline Ponto $4(20-40 \mathrm{~cm})$ & 6,1 & 16,5 & 21 & 0,11 & 6,23 & 0,42 & 4,8 & 0 & 11,55 & 0 & 59 \\
\hline Ponto $5(0-20 \mathrm{~cm})$ & 8,2 & 18 & 89 & 0,43 & 9,54 & 0,43 & 0,3 & 0 & 10,66 & 0 & 98 \\
\hline Ponto $5(20-40 \mathrm{~cm})$ & 8,4 & 11,9 & 23 & 0,34 & 10,21 & 0,39 & 0 & 0 & 10,87 & 0 & 100 \\
\hline Ponto $6(0-20 \mathrm{~cm})$ & 6,8 & 8,2 & 3 & 0,09 & 4,96 & 0,25 & 2,6 & 0 & 7,86 & 0 & 67 \\
\hline Ponto $6(20-40 \mathrm{~cm})$ & 6,8 & 6,8 & 2 & 0,09 & 3,26 & 0,21 & 2,4 & 0 & 6 & 0 & 59 \\
\hline Ponto $7(0-20 \mathrm{~cm})$ & 5,5 & 5,3 & 6 & 0,24 & 4,7 & 0,23 & 5,7 & 0,69 & 10,91 & 11 & 47 \\
\hline Ponto $7(20-40 \mathrm{~cm})$ & 5,3 & 7,4 & 4 & 0,11 & 3,08 & 0,18 & 7,5 & 1,91 & 10,83 & 36 & 31 \\
\hline Ponto $8(0-20 \mathrm{~cm})$ & 5,3 & 3,1 & 2 & 0,16 & 3,6 & 0,41 & 6,8 & 4 & 11 & 49 & 38 \\
\hline Ponto $8(20-40 \mathrm{~cm})$ & 5,1 & 5,2 & 2 & 0,14 & 1,59 & 0,24 & 9,2 & 6,87 & 11,17 & 78 & 18 \\
\hline
\end{tabular}

pH em água 1:2:5; M.O. por digestão úmida, P e K determinados pelo método Mehllich I; Ca, Mg e Al trocáveis extraídos com KCl $1 \mathrm{~mol} / \mathrm{L}$.

Fonte: Laudo de análise de solo realizado pela EMBRAPA em 07/01/2016.

TABELA 4. Classes de interpretação para a acidez ativa do solo $(\mathrm{pH})^{1}$ Classificação agronômica ${ }^{2}$

\begin{tabular}{ccccc}
\hline Muito Baixo & Baixo & Bom & Alto & Muito Alto \\
\hline$<4,5$ & $4,5-5,4$ & $5,5-6,0$ & $6,1-7,0$ & $>7,0$ \\
\hline 1
\end{tabular}

${ }_{1}^{1} \mathrm{pH}$ em $\mathrm{H}_{2} \mathrm{O}$, relação 1:2,5, TFSA: $\mathrm{H}_{2} \mathrm{O} .{ }^{2} \mathrm{~A}$ qualificação utilizada indica adequado (Bom) ou inadequado (muito baixo e baixo ou alto e muito alto).

Fonte: (RIBEIRO et al., 1999). 
TABELA 5. Classes de interpretação de fertilidade de solo para a matéria orgânica e para o complexo de troca catiônica.

\begin{tabular}{|c|c|c|c|c|c|c|}
\hline \multirow{2}{*}{ Característica } & \multirow{2}{*}{ Unidade } & \multicolumn{5}{|c|}{ Classificação } \\
\hline & & Muito baixo & Baixo & Médio $^{1}$ & Bom & Muito bom \\
\hline \multirow{7}{*}{$\begin{array}{c}\text { M.O } \\
\text { P } \\
\text { K } \\
\text { Ca } \\
\text { Mg } \\
\text { CTC } \\
\text { V }\end{array}$} & \multirow{7}{*}{$\begin{array}{c}\mathrm{g} \mathrm{kg}^{-1} \\
\mathrm{mg}^{-\mathrm{dm}^{-3}} \\
\mathrm{cmol}_{\mathrm{c}} \mathrm{dm}^{-3} \\
\mathrm{cmol}_{\mathrm{c}} \mathrm{dm}^{-3} \\
\mathrm{cmol}_{\mathrm{C}} \mathrm{dm}^{-3} \\
\mathrm{cmol}_{\mathrm{c}} \mathrm{dm}^{-3} \\
\%\end{array}$} & $\leq 7,0$ & $7,1-20,0$ & $20,1-40,0$ & $40,1-70,0$ & $>70,0$ \\
\hline & & $\leq 6,6$ & $6,7-12,0$ & $12,1-20,0$ & $20,1-30,0$ & $>30,0$ \\
\hline & & $\leq 0,040$ & $0,05-0,10$ & $0,11-0,18$ & $0.19-0,31$ & $>0,31$ \\
\hline & & $\leq 0,40$ & $0,41-1,20$ & $1,21-2,40$ & $2,41-4,00$ & $>4,00$ \\
\hline & & $\leq 0,15$ & $0,16-0,45$ & $0,46-0,90$ & $0,91-1,50$ & $>1,50$ \\
\hline & & $\leq 1,60$ & $1,61-4,30$ & $4,31-8,60$ & $8,61-15,00$ & $>15,00$ \\
\hline & & $\leq 20,0$ & $20,1-40,0$ & $40,1-60,0$ & $60,1-80,0$ & $>80,0$ \\
\hline \multirow{5}{*}{$\begin{array}{c}\mathrm{H}+\mathrm{Al} \\
\mathrm{Al} \\
\mathrm{m}\end{array}$} & \multirow{5}{*}{$\begin{array}{c}\mathrm{Cmol}_{\mathrm{C}} \mathrm{dm}^{-3} \\
\mathrm{cmol}_{\mathrm{C}} \mathrm{dm}^{-3} \\
\%\end{array}$} & \multicolumn{5}{|c|}{ Classificação } \\
\hline & & Muito baixo & Baixo & Médio ${ }^{2}$ & Alta & Muito alta \\
\hline & & $\leq 1,00$ & $1,01-2,50$ & $2,51-5,00$ & $5,01-9,00$ & $>9,00$ \\
\hline & & $\leq 0,21$ & $0,21-0,50$ & $0,51-1,00$ & $1,01-2,00$ & $>2,00$ \\
\hline & & $\leq 15,0$ & $15,1-30,0$ & $30,1-50,0$ & $50,1-75,0$ & $>75,0$ \\
\hline
\end{tabular}

${ }^{1} \mathrm{O}$ limite superior desta classe indica o nível crítico, ou seja, indica o teor do elemento no solo abaixo do qual a produção é limitada.

2 O limite superior desta classe indica o nível crítico, ou seja, indica o teor do elemento no solo acima do qual a produção é limitada.

M.O. (método Walkley \& Black). P e K (método Mehlich-1). Ca, $\mathrm{Mg}$ e Al (método $\mathrm{KCl} 1 \mathrm{~mol} / \mathrm{L}$ ). CTC ou $\mathrm{T}\left(\mathrm{SB}+(\mathrm{H}+\mathrm{Al}) . \mathrm{V} \%(100 \times \mathrm{SB} / \mathrm{T}) \cdot \mathrm{H}+\mathrm{Al}\right.$ (Método $\left.\mathrm{Ca}(\mathrm{OAc})_{2} 0,5 \mathrm{~mol} / \mathrm{L}, \mathrm{pH} 7\right) \cdot \mathrm{m} \%\left(100 \mathrm{Al}^{3+} / \mathrm{t}\right)$.

Fonte: Adaptado de RIBEIRO et al., (1999).

\section{Interpretação das análises de fertilidade do solo nos 8 pontos considerando a profundidade de $0-20 \mathrm{~cm}$}

Para facilitar a interpretação das análises de fertilidade do solo optou-se por sistematizar a comparação dos resultados encontrados em campo $(0-20 \mathrm{~cm}) \mathrm{com} o$ adequado para cada característica de fertilidade (Tabela 6).

TABELA 6. Interpretação dos resultados da análise de fertilidade do solo para a profundidade de $0-20 \mathrm{~cm}$ nos oito pontos de estudos na APP do Igarapé dos Tanques presentes na tabela 3 , com base nos valores adequados ao desenvolvimento de espécies arbóreas nativas expostos nas tabelas 4 e 5.

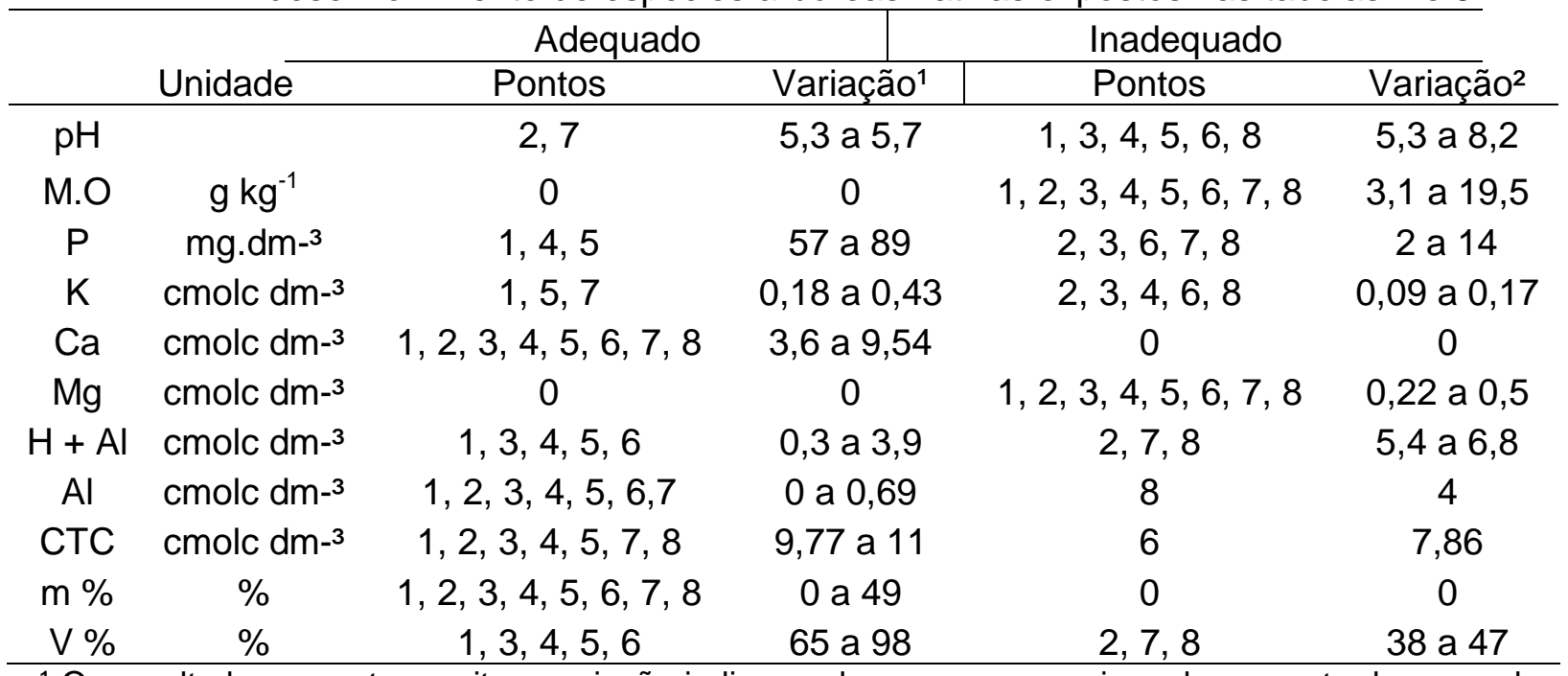

${ }^{1}$ Os resultados expostos no item variação indica qual o menor e o maior valor encontrado em cada característica nos pontos em que as mesmas se encontram adequadas.

2 Os resultados expostos no item variação indica qual o menor e o maior valor encontrado em cada característica nos pontos em que as mesmas se encontram inadequadas. 
Em relação à matéria orgânica (M.O) do solo, todos os oito pontos avaliados apresentaram teores inadequados. Tendo em vista a importância da M.O, principalmente para a melhoria das propriedades físicas e biológicas do solo, recomenda-se o uso de práticas agrícolas que proporcionem o aumento deste elemento no solo da área de estudo. Entre estas práticas pode ser utilizado esterco animal, restos de culturas, adubos verdes etc.

Quanto aos elementos $\mathrm{P}$ e K, dos oito pontos avaliados, 5 pontos apresentaram teores inadequados. Nestes pontos se faz necessário a reposição desses elementos por meio do uso de fertilizantes.

A respeito dos teores de $\mathrm{Ca}$ e $\mathrm{Mg}$, todos os oito pontos apresentaram teores adequados para o $\mathrm{Ca}$ e teores inadequados para $\mathrm{Mg}$. Diante do exposto, nos pontos que necessitarem de calagem, deve-se optar por calcário do tipo que apresenta maiores teores de $\mathrm{Mg}$, já nos pontos onde não há a necessidade de calagem, devese realizar adubação magnesiana.

Em relação ao $\mathrm{H}+\mathrm{Al}$, dos oito pontos avaliados, 3 pontos apresentaram valores inadequados. Quanto ao $\mathrm{Al}$, dos oito pontos avaliados, apenas 1 ponto apresentou valor inadequado. A respeito da saturação por alumínio (m\%), todos os oito pontos apresentaram valores adequados.

Para o limite crítico da saturação de bases (V\%), abaixo do qual o desenvolvimento das plantas é limitado, utilizou-se o valor de $50 \%$, conforme estudo realizado por VAN RAIJ et al. (1997) para áreas de reflorestamento misto. Utilizandose deste parâmetro, o V\% apresentou valores inadequados em 3 dos oito pontos analisados, o que indica a necessidade de calagem nos referidos pontos. Ao realizar calagem nos pontos inadequados, além de corrigir o $\mathrm{V} \%$, provavelmente o $\mathrm{H}+\mathrm{Al}$ e o $\mathrm{m} \%$ também serão corrigidos, já que a prática de calagem eleva os valores de $\mathrm{V} \% \mathrm{e}$ diminui os de $\mathrm{H}+\mathrm{Al}$ e de $\mathrm{m} \%$.

Quanto à capacidade de troca catiônica (CTC), dos oito pontos avaliados, apenas 1 ponto apresentou valor inadequado. A elevação da CTC pode ser realizada com o uso de práticas que contribuem para o aumento do teor de matéria orgânica neste ponto.

\section{Interpretação das análises de fertilidade do solo nos oito pontos considerando a profundidade de $20-\mathbf{4 0} \mathrm{cm}$}

Com relação à interpretação das análises realizadas na profundidade de 20$40 \mathrm{~cm}$, estas servem para diagnosticar possíveis condições desfavoráveis ao desenvolvimento radicular. Tais condições são identificadas pelos valores de $\mathrm{Ca}$ menor que $0,4 \mathrm{cmol}_{\mathrm{c}} \mathrm{dm}^{3}$ e Al maior que $0,5 \mathrm{cmol}_{\mathrm{c}} \mathrm{dm}^{3}$, associado com saturação por alumínio (m\%) maior que $40 \%$ (VAN RAIJ et al., 1997).

Em nenhum dos pontos analisados na APP do Igarapé dos Tanques foi apresentado valores de cálcio ( $\mathrm{Ca}$ ) na profundidade de $20-40 \mathrm{~cm}$ abaixo do recomendado por VAN RAIJ et al. (1997). Quanto ao alumínio (Al) associado à saturação por alumínio (m\%), apenas o ponto 8 apresentou valor superior ao nível crítico acima do qual o desenvolvimento das espécies arbóreas nativas destinadas a reflorestamento misto é prejudicado. Diante do exposto, todos os oito pontos analisados apresentaram condições favoráveis ao desenvolvimento radicular na profundidade de $20-40 \mathrm{~cm}$, não necessitando, portando, adubação e/ou calagem nesta profundidade.

A variação nos valores dos nutrientes do solo da área de APP do lgarapé dos Tanques era prevista, tendo em vista a heterogeneidade do solo ocasionada pela 
constante influência antrópica dentro do perímetro urbano da cidade de Porto Velho $-\mathrm{RO}$.

\section{CONCLUSÃO}

Os resultados obtidos em campo permitiram concluir que:

1. Os valores de resistência do solo à penetração nas 4 profundidades $(0-10,10$ $20,20-30$ e $30-40 \mathrm{~cm}$ ) dos oito pontos avaliados estão acima do valor de 2,0 MPa, o que indica condição de compactação no solo e consequente prejuízo ao desenvolvimento de raízes de espécies arbóreas nativas destinadas à recuperação da APP do Canal dos Tanques, sendo que os pontos que apresentaram as maiores resistências foram os pontos 2 e 3 nas profundidades de $20-30$ e $30-40 \mathrm{~cm}$ e o ponto 6 nas profundidades de $10-20,20-30$ e $30-40 \mathrm{~cm}$.

2. Em relação a fertilidade do solo, para a camada de $0-20 \mathrm{~cm}$, todos os oito pontos apresentaram valores adequados para o Ca. Quanto aos demais parâmetros recomendados para adubação, os oito pontos apresentaram valores inadequados de um ou mais elementos. Na camada de $20-40 \mathrm{~cm}$, todos os oito pontos apresentaram condições favoráveis ao desenvolvimento radicular de espécies arbóreas nativas, não necessitando, assim, adubação e/ou calagem para esta profundidade.

3. A heterogeneidade nos valores de resistência à penetração e de fertilidade do solo refletem o quanto a APP do Igarapé dos Tanques está sob influência antrópica. Tais condições podem ser consideradas como fatores de limitação para a recuperação dessa área.

\section{REFERÊNCIAS}

ASSIS, E. B. de; NUNES, M. C. M.; BARELLI, M. A. A.; SEABRA JUNIOR, S.; SERAFIM, M. E. Resistência à penetração em argissolo vermelho-amarelo sob pousio e diferentes culturas de cobertura. Enciclopédia Biosfera, Centro Científico Conhecer - Goiânia, v. 10, n. 19, p.1678-1677, 2014. Disponível em : < http://www.conhecer.org.br/enciclop/2014b/AGRARIAS/resistencia\%20a\%20penetra cao.pdf >

BENEDETTI, M. M.; DUARTE, I. N.; MELO JUNIOR, H. B. de; BORGES, E. N. Resistência do solo à penetração em um latossolo vermelho distrófico típico sob diferentes usos. Enciclopédia Biosfera, Centro Cientifico Conhecer - Goiânia, v. 6, n. 11; p.1-9, 2010. Disponível em: < http://www.conhecer.org.br/enciclop/2010c/resistencia\%20do\%20solo.pdf >

BENGHOUGH, A.G.; MULLINS, C.E. Mechanical impedance to root growth: a review of experimental techniques and root growth responses. Journal of Soil Science, v.41, p.341-358, 1990.

BRASIL. Lei Complementar no LEI № 12.651, DE 25 DE MAIO DE 2012. Disponível em: < http://www.planalto.gov.br/ccivil_03/_ato2011-2014/2012/lei/l12651.htm > 
CAMPOS, M. C. C; SOARES, M. D. R.; AQUINO, R. E.; SANTOS, L. A. C.; MANTOVANELLI, B. C. Distribuição espacial da resistência do solo à penetração e teor de água do solo em uma área de agrofloresta na região de Humaitá, AM. Comunicata Scientiae, v. 5 , n. 4, out-dez, p. 509-517, 2014. Bom Jesus, Piauí. Disponível em: http://comunicatascientiae.com.br/comunicata/article/view/307/293

CONTE, O.; FLORES, J. P. C.; CASSOL, L. C.; ANGHINONI, I.; CARVALHO, P. C. de F.; LEVIEN, R.; WESP, C. de L. Evolução de atributos físicos de solo em sistema de integração lavoura-pecuária. Pesquisa Agropecuária Brasileira, v.46, n.10, out. p.1301-1309, 2011. Brasília, Brasil. Disponível em: < http://seer.sct.embrapa.br/index.php/pab/article/view/8958/6638 > doi: <http://dx.doi.org/10.1590/S0100-204X2011001000026>

EMPRESA BRASILEIRA DE PESQUISA AGROPECUÁRIA - Embrapa. Manual de métodos de análise de solos. 2 ed. Rio de Janeiro: Embrapa Solos, 230p., 2011. Disponível em: <http://www.geografia.fflch.usp.br/graduacao/apoio/Apoio/Apoio_Attila/2s2015/excur soes/Manual-de-Metodos-de-Analise-de-Solo_EMBRAPA_2011.pdf >

FERNANDES, L. C.; GUIMARÃES, S. C. P. (Coord.). Atlas Geoambiental de Rondônia. Porto Velho: SEDAM, 2001.

GUBIANI, P. I.; REICHERT, J. M.; REINERT, D. J. Indicadores hídrico-mecânicos de compactação do solo e crescimento de plantas. Revista Brasileira de Ciência do Solo, v. 37, n. 1, enero-febrero, p. 1-10, 2013. Sociedade Brasileira de Ciência do Solo, Viçosa, Brasil. Disponível em: <http://www.redalyc.org/articulo.oa?id=18022573602> doi: <http://dx.doi.org/10.1590/S0100-06832013000100001>

GUBIANI, P. I.; REICHERT, J. M.; REINERT, D. J.. Interação entre disponibilidade de água e compactação do solo no crescimento e na produção de feijoeiro. Revista Brasileira de Ciência do Solo, v. 38, n. 3, maio-jun, p. 765-773, 2014. Sociedade Brasileira de Ciência do Solo, Viçosa, Brasil. Disponível em: <http://www.sbcs.org.br/wp-content/uploads/2014/07/V38N3a08.pdf> doi: <http://dx.doi.org/10.1590/S0100-06832014000300008>

KUNZ, M..; GONÇALVES, A. D. M. de A.; REICHERT, J. M.; GUIMARÃES, R. M. L.; REINERT, D. J.; RODRIGUES, M. F. Compactação do solo na integração sojapecuária de leite em Latossolo argiloso com semeadura direta e escarificação. Revista Brasileira de Ciência do Solo, v. 37, n. 6, p.1699-1708, 2013. Sociedade Brasileira de Ciência do Solo, Viçosa, Brasil. Disponível em: http://www.scielo.br/scielo.php?script=sci_arttext\&pid=S0100-

06832013000600026\&lng=pt\&nrm=iso\&tIng=en doi: <http://dx.doi.org/10.1590/S0100-06832013000600026>

LIMA, R. P. de; LEÓN, M. J. de; SILVA, A. R. da. Compactação do solo de diferentes classes texturais em áreas de produção de cana-de-açúcar. Revista Ceres, v. 60, n. 1, jan-fev, p. 016-020, 2013. Viçosa, Brasil. Disponível em: < http://www.ceres.ufv.br/ojs/index.php/ceres/article/view/3939/1776> doi: $<$ http://dx.doi.org/10.1590/S0034-737X2013000100003> 
LIMA, R. P. de; SILVA, A. R. da; RAMINELLI, J. A. Importância de atributos físicos do solo na identificação da compactação. Revista Verde de Agroecologia e Desenvolvimento Sustentável, v. 8, n. 1, jan-mar, p. 93-97, 2013. Mossoró - RN, Brasil.

em:

<http://gvaa.org.br/revista/index.php/RVADS/article/view/1852/1512 >

ORTIGARA, C.; KOPPE, E.; LUZ, F. B. da; BERTOLLO, A. M.; KAISER, D. R.; SILVA, V. R. da. Uso do solo e propriedades físico-mecânicas de Latossolo Vermelho. Revista Brasileira de Ciência do Solo, v. 38, n. 2, mar-abr, p.619-626, 2014. Sociedade Brasileira de Ciência do Solo, Viçosa, Brasil. Disponível em: < http://www.scielo.br/scielo.php?script=sci_arttext\&pid=S0100-

06832014000200026\&lng=pt\&nrm=iso\&tIng=en>

doi:

$<$ http://dx.doi.org/10.1590/S0100-06832014000200026>

RIBEIRO, A. C.; GUIMARÃES, P. T. G.; ALVAREZ V. V. H. (Eds). Recomendações para o uso de corretivos e fertilizantes em Minas Gerais-5a Aprox. Viçosa, MG, Comissão de Fertilidade do Solo do Estado de Minas Gerais. 359p.: il., 1999.

RONDÔNIA. Secretaria de Estado do Desenvolvimento Ambiental - SEDAM. Porto Velho, Rondônia, 2015.

SALMAN, A. K. D.; LÓPEZ, G. F. Z.; BENTES-GAMA, M. M.; ANDRADE, C. M. S. Espécies arbóreas nativas da Amazônia Ocidental Brasileira com potencial para arborização de pastagens. Embrapa, documentos, 127, 20p., Porto Velho, RO: Embrapa Rondônia, 2008.

SANTANA, A. V. de; FERREIRA, E. de M.; GUIMARÃES, L. E.; CALIL, F. N.; TSAI, H. U. Resistência à penetração em solo sob sistema integração lavoura-pecuáriafloresta, pasto convencional e mata nativa do cerrado. Enciclopédia Biosfera, Centro Científico Conhecer - Goiânia, v. 10, n .18, p.2675-2680, 2014. Disponível em: < http://www.conhecer.org.br/enciclop/2014a/AGRARIAS/resistencia.pdf >

SILVA, F. A. S. ASSISTAT: Versão 7.7 beta. DEAG-CTRN-UFCG - Atualizado em 01 de janeiro de 2016. Disponível em <http://www.assistat.com/>. Acessado em: 06 de março de 2016.

STOLF, R.; FERNANDES, J.; FURLANI NETO, V. L. Recomendação para uso do penetrômetro de impacto, modelo IAA/Planalsucar - Stolf. São Paulo, MIC/IAA/PNMCA-Planalsucar, 1983. 8p. (Série penetrômetro de impacto-Boletim, 1).

STOLF, R. Penetrômetro de Impacto Stolf- programa de manipulação de dados em Excel-VBA. UFSCar, $2011 . \quad$ Disponível em: <http://www.cca.ufscar.br/drnpa/hprubismar.htm>

VAN RAIJ, B. CANTARELA, H., QUAGGIO, J. A., FURLANI, A. M. C. Recomendações de adubação e calagem para o Estado de São Paulo. 2.ed. ver. atual. Campinas, Instituto Agronômico de Campinas / Fundação IAC, 285p. Boletim Técnico, 100, 1997. 